\section{In a spin}

Assorted arrays

The PathwayFinder gene arrays from SuperArray Bioscience are designed to help you study relative gene expression in new experimental systems. They contain representative genes from several different signal transduction or cancer pathways. Changes in the expression of these key genes indicate the activation or suppression of those pathways. The company says these arrays allow researchers to rapidly assess which pathways are affected by certain experimental conditions. Researchers can then perform a comprehensive survey of gene expression within those pathways using the company's other application-specific cDNA arrays. The PathwayFinder product line includes Signal Transduction PathwayFinder, GProtein Coupled Receptor Signaling PathwayFinder, Calcium/cAMP Signaling PathwayFinder, Cancer PathwayFinder I,
Cancer PathwayFinder II, Stress and Toxicity PathwayFinder. www.superarray.com

Monitor four cardiac markers simultaneously with the new antibody array from Panomics. The stated benefits of the TranSignal Cardiac Marker Array are high sensitivity (the array can detect cardiac markers in the same range as ELISAs), ease of use, and the choice of two detection systems (chemiluminescent and colorimetric). With the array it is possible to detect cardiac markers - creatine kinase MB, high sensitivity C-reactive protein, myoglobin and cardiac troponin I - in plasma or sera. Tel. (+1) 877-726-6642 (USA and Canada) www.panomics.com

\section{Detection kits}

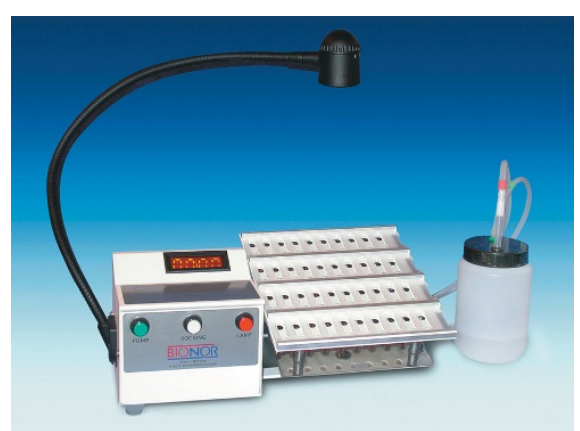

Bionor testing station

Bionor has developed a simple and semirapid test for confirming HIV-1 and -2 serological status. The Bionor HIV 1\&2 Confirmatory Test is based on an ELISA principle using magnetic beads as a solid support where antibody responses to individual viral proteins are visualized separately. Purified peptides to HIV-1 core p24 ${ }^{\mathrm{CA}}$, HIV-1 transmembrane glycoproteins gp $41^{\mathrm{TM}}$, HIV-1 envelope glcycoproteins gp $160^{\mathrm{ENV}}$ and HIV-2 transmembrane glycoporteins $\mathrm{gp} 36^{\mathrm{TM}}$ are used as a source of antigen. The company says the results are interpreted easily and serological status is determined according to the CDC/ASTPHLD guidelines. The use of peptides as a source of antigen is said to minimize non-specific responses that can often complicate the interpretation of western blot assays normally used to confirm HIV serological status. Bionor says its HIV 1\&2 Confirmatory Test demonstrates an equivalent sensitivity to current western blot assays and provides greater specificity due to fewer indeterminate results. By combining the Bionor rapid HIV
1\&2 Screening Test and HIV 1\&2 Confirmatory Test, HIV serological status can be both detected and confirmed within hours. All reagents including pipetting aids, as well as both positive and negative controls, are supplied in the kit, which is used in conjunction with the Bionor Testing Station. This consists of a rocking platform with magnets, aspirator, lamp and waste container.

www.bionor.no

\section{Mail-order means}

Bender MedSystems has issued a product guide for its line of immunology and cell biology research products. The guide is divided into 12 major product categories and provides over 150 ELISA and Instant ELISA kits for work in cell adhesion, apoptosis, cytokine/growth factors and tumor biology. It also includes a wide selection of module sets, sample diluents, fluorescent bead immunoassays, antibodies, proteins, BioLISAs, ELISpots and transferrinfection products, as well as intrastain cell fixation and permeabilization kits.

Tel. (+43) 1-796-40-40-0

www.bendermedsystems.com

\section{Improving your image}

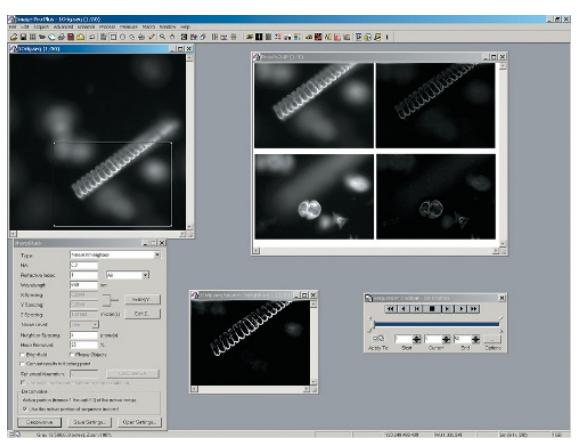

Picture perfect-see Media Cybernetics

Media Cybernetics announces an upgrade release of SharpStack. This plug-in module for Image-Pro Plus allows microscopists to obtain clear images from a stack of hazy images. Unlike other deconvolution solutions, the company says that SharpStack integrates seamlessly with image capture, two-dimensional processing, analysis, and reporting within Image-Pro Plus. SharpStack's algorithm options include nearest neighbor, no neighbor, inverse filter and spherical aberration correction.

www.mediacy.com 